\title{
Diretrizes de acessibilidade de interfaces digitais para pessoas com Transtorno do Espectro Autista: uma revisão integrativa de literatura
}

\author{
Digital interface accessibility guidelines for people with Autism Spectrum \\ Disorder: an integrative literature review
}

Directrices de accesibilidad de la interfaz digital para personas con Trastorno del Espectro Autista: una revisión integradora de literatura

\author{
Andiara Cristina de Souza \\ Doutoranda na Universidade Federal de São Carlos, São Carlos, SP, Brasil \\ E-mail: ancrisou@gmail.com ORCID: https://orcid.org/0000-0003-0160-6488 \\ Priscila Benitez \\ Professora doutora da Universidade Federal do ABC, Santo André, SP, Brasil \\ E-mail: priscila.benitez@ufabc.edu.br ORCID: https://orcid.org/0000-0003-3501-7606 \\ João dos Santos Carmo \\ Professor doutor da Universidade Federal de São Carlos, São Carlos, SP, Brasil \\ E-mail: jcarmo@ufscar.br ORCID: https://orcid.org/0000-0003-3913-7023
}

Recebido em 04 de novembro de 2020

Aprovado em 26 de maio de 2021

Publicado em 21 de junho de 2021

\section{RESUMO}

Estudos evidenciaram que pessoas com TEA apresentam grande afinidade e interesse por recursos tecnológicos. Sites, aplicativos e softwares destinados a estes usuários têm sido desenvolvidos em grande escala. Todavia, pouco se sabe sobre suas interfaces e se são desenvolvidas com base em evidências empiricamente testadas e que assegurem a sua acessibilidade. Neste sentindo, a identificação e análise de diretrizes de acessibilidade podem fazer com que as interfaces tecnológicas sejam mais acessíveis a esta camada da população. $O$ objetivo deste trabalho foi mapear na literatura pesquisas empíricas que avaliaram diretrizes para acessibilidade digital envolvendo pessoas com TEA. Trata-se de uma revisão integrativa pautada no protocolo PRISMA. A partir dos critérios de exclusão e com base na análise do título e resumo, foram designados, para compor o corpus final de análise, sete artigos. Os resultados apresentados evidenciam que ainda existe uma lacuna importante na literatura no que diz respeito à avaliação de diretrizes de acessibilidade digital para pessoas com TEA. Constatou-se que os poucos estudos empíricos experimentais existentes focam em adultos com TEA leve, o que torna muito difícil alcançar generalizações. Apesar de representar um passo importante no caminho da inclusão digital de pessoas com TEA, as diretrizes de acessibilidade ainda caminham de maneira tímida e carecem de pesquisas empíricas e testagens. 
http://dx.doi.org/10.5902/1984686X62649

Palavras-chave: Diretrizes de acessibilidade; Transtorno do Espectro Autista; Tecnologia Digital.

\section{ABSTRACT}

Studies show that people with ASD have a great affinity and interest in technological resources. Sites, applications, and software services to these users have been developed on a large scale. However, little is known about their interfaces and whether they are developed based on empirically tested evidence that ensures their accessibility. In this sense, identification and analysis of accessibility guidelines can make technological interfaces return more to this population layer. This work's objective was to map empirical research in the literature that evaluated guidelines for digital accessibility involving people with ASD. This research is an integrative review based on the PRISMA protocol. Based on the exclusion criteria and based on the title and abstract analysis, seven articles were designated to compose the final corpus of analysis. The results show that there is still an important gap in the literature regarding assessing digital accessibility guidelines for people with ASD. It was found that the few existing experimental empirical studies focus on adults with high functioning ASD or with mild impairment, which leads to the impossibility of generalizations. Despite representing a critical step in the digital inclusion of people with ASD, accessibility guidelines still walk timidly away and lack empirical research and testing.

Keywords: Accessibility guidelines; Autistic Spectrum Disorder; Digital Technology.

\section{RESUMEN}

Los estudios demuestran que las personas con TEA presentan una gran afinidad e interés por los recursos tecnológicos. Los sitios, aplicaciones y servicios de software para estos usuarios se han desarrollado a gran escala. Sin embargo, se sabe poco sobre sus interfaces y si se desarrollan con base en evidencia probada empíricamente que asegura su accesibilidad. En este sentido, una identificación y análisis de las pautas de accesibilidad pueden hacer que las interfaces tecnológicas vuelvan más a este segmento de la población. El objetivo de este trabajo fue mapear la investigación empírica en la literatura que evaluó las pautas de accesibilidad digital involucrando a personas con TEA. Esta es una revisión integradora basada en el protocolo PRISMA. Con base en los criterios de exclusión y en base al análisis del título y resumen, se designaron siete artículos para componer el corpus final de análisis. Los resultados obtenidos muestran que aún existe una laguna importante en la literatura en cuanto a la evaluación de las pautas de accesibilidad digital para personas con TEA. Se encontró que los pocos estudios empíricos experimentales existentes se enfocan en adultos con TEA de alto funcionamiento 0 con deterioro leve, lo que conduce a la imposibilidad de generalizaciones. A pesar de representar un paso importante en el camino de la inclusión digital de las personas con TEA, las pautas de accesibilidad aún caminan de manera tímida y carecen de investigación y pruebas empíricas.

Palabras clave: Pautas de accesibilidad; Trastorno del espectro autista; Tecnología Digital. 
http://dx.doi.org/10.5902/1984686X62649

\section{Introdução}

O Transtorno do Espectro Autista (TEA) se classifica como um conjunto específico de déficits comportamentais qualitativos no desenvolvimento sociocomunicativo, comportamentos estereotipados, com repertório restrito de interesses e atividades. Segundo o DSM-V, o TEA é classificado como transtorno do neurodesenvolvimento (APA, 2014). Este conjunto de sintomas pode limitar ou dificultar o funcionamento diário do indivíduo, ocasionando prejuízos de ordem pessoal, social, acadêmica e profissional (APA, 2014).

Estas características comuns, entretanto, não podem ser utilizadas de modo a uniformizar as pessoas com TEA dentro de um grupo homogêneo. Em virtude de tratar-se de um espectro, o autismo apresenta uma grande diversidade de características e graus de comprometimento, podendo este ir de leve a grave. O TEA pode, ainda, estar associado a outras comorbidades, comumente relacionadas à Deficiência Intelectual (APA, 2014).

Por meio do apoio familiar e uma ação interventiva multiprofissional, é possível promover melhoras significativas na vida da pessoa com TEA (LOCATELLI, 2016), como, por exemplo, por meio da intervenção comportamental intensiva, identificada na revisão de Medavarapu et al. (2019) como tratamento comprovadamente científico.

Para a devida implementação da intervenção comportamental, é necessário a avaliação do repertório comportamental, registro e análise dos dados para posterior tomada de decisão, em relação aos objetivos de ensino de cada comportamento específico, em cada área do desenvolvimento. Nesse caso, o uso de tecnologia tem sido valioso, do ponto de vista de otimizar o registro dos dados e análise, assim como no fornecimento de instruções curtas e padronizadas (SILVA; SOARES; BENITEZ, 2020; TREVISAN et al., 2019).

Ao longo das duas últimas décadas, as tecnologias digitais têm sido utilizadas como suporte para pais e terapeutas, buscando facilitar o aprendizado e a independência dos indivíduos com TEA (CHIEN et al., 2015), principalmente como favorecedoras para garantia da quantidade mínima de horas da intervenção comportamental intensiva, assim como para avaliação da generalização da aprendizagem (SILVA; SOARES; BENITEZ, 2020; TREVISAN et al., 2019). Portanto, desenvolver tecnologias digitais para otimização das intervenções comportamentais pode ser um passo importante para garantir um maior envolvimento de pessoas, por meio da escalabilidade, já que, no Brasil, não existe uma normativa na área da saúde, educação e assistência social que garanta esse tipo de 
http://dx.doi.org/10.5902/1984686X62649

intervenção no contexto das políticas públicas. Alternativas como o uso das tecnologias digitais podem criar condições para favorecer o envolvimento de pessoas que não teriam condições se dependessem unicamente de verba privada.

Ao analisar as tecnologias digitais disponíveis, é possível constatar que grande parte das diretrizes utilizadas para a produção de softwares e aplicativos tem uma linguagem voltada para as pessoas da área computacional, o que torna sua compreensão difícil para pessoas leigas nesta área (DAREJEH; SINGH, 2013; PAVLOV, 2014; BRITTO; PIZZOLATO, 2016; REZAE et al., 2020; SILVA; SOARES; BENITEZ, 2020). A fim de tornar as interfaces digitais mais acessíveis e facilitar a sua utilização por diferentes tipos de usuários, é necessário o desenvolvimento de diretrizes que visem atender a diferentes especificidades dos usuários, tornando os sistemas mais personalizáveis e interativos, levando em conta uma perspectiva do desenho universal (BRASIL, 2016).

Prever, portanto, condições de acessibilidade para todas as pessoas, desde o design da tecnologia até sua efetiva implementação, é fundamental para o desenvolvimento de qualquer tecnologia digital, sobretudo na área educacional inclusiva, para garantir o envolvimento de alunos com TEA. Para propósitos deste estudo, será utilizado o termo "acessibilidade" no sentido de flexibilidade proporcionada para o acesso à informação e à interação, de maneira que usuários com diferentes necessidades possam acessar e usar esses sistemas (SILVA; BARBOSA, 2010, p. 32).

A acessibilidade, em termos digitais, possibilita que as interfaces se tornem operáveis e compreensíveis por uma ampla variedade de pessoas com diversas habilidades e em diferentes condições ambientais e de estímulos, ampliando a interatividade com o software (HENRY, 2007). Interfaces acessíveis possibilitam maior interação do usuário com o software, contribuindo para melhor aproveitamento de seus benefícios. Por outro lado, interfaces complexas e difíceis de usar podem confundir os usuários, levando-os a abandonar o software mesmo que este the traga benefícios (DAREJEH; SINGH, 2013; PAVLOV, 2014; BRITTO; PIZZOLATO, 2016; REZAE et al., 2020).

Com a finalidade de tornar websites acessíveis a todos, a World Wide Web Consortium (W3C) ${ }^{1}$, a partir de meados da década de 1990, tem desenvolvido diretrizes de acessibilidade, também chamadas de guidelines. Trata-se de um conjunto de recomendações/orientações não obrigatórias que buscam direcionar 0 trabalho de desenvolvedores (W3C, 2005). A Web Accessibility Initiative (WAI) é um dos braços do W3C, sendo o órgão responsável por desenvolver diretrizes, estratégias e recursos para 
http://dx.doi.org/10.5902/1984686X62649

acessibilidade da web. Dentre elas, está a WCAG - Web Content Accessibility Guidelines, que, atualmente, se encontra na versão 2.1 e traz algumas recomendações relacionadas a deficiências cognitivas. Estas diretrizes visam diminuir as barreiras para que pessoas com diferentes deficiências possam acessar a internet.

As guidelines buscam realizar uma padronização para elaboração de ambientes informacionais digitais, objetivando solucionar problemas que possam influir negativamente na acessibilidade, privando os usuários do acesso ao conteúdo de um determinado site. Apesar de não obrigatórias, estas diretrizes são importantes para o processo de tomada de decisão ao implementarem uma determinada solução (BRITTO; PIZZOLATO, 2016).

Estudos sobre acessibilidade têm sido desenvolvidos ao longo das últimas décadas. Todavia, grande parte deles, conforme destacam Britto e Pizzolato (2016) e Eraslan et al. (2018), tem como foco deficiências físicas, visuais e auditivas, havendo uma importante lacuna no que diz respeito às deficiências cognitivas. Reconhecendo esta lacuna, o W3C criou no ano de 2014 um grupo de força-tarefa denominado Cognitive and Learning Disabilities Task Force (COGA), com a finalidade de desenvolver pesquisas relacionadas à acessibilidade web para pessoas com deficiências cognitivas, neuronais ou de aprendizagem (SEEMAN; COOPER, 2016). Ao pensar em um público heterogêneo, é possível propor soluções diversificadas que atendam a um maior número de pessoas, independentemente de suas condições, conforme previsto no desenho universal (BRASIL, 2016).

No ano de 2016, o COGA publicou algumas diretrizes gerais para o desenvolvimento de sites para usuários com deficiências cognitivas, neuronais ou de aprendizagem. As orientações eram divididas em categorias e algumas se relacionavam especificamente com TEA.

Tendo como uma das referências o COGA e sem buscar substituir as normas da WCAG, Britto e Pizzolato (2016) apresentaram, no Brasil, um conjunto de diretrizes de acessibilidade voltadas para usuários com TEA, denominado Guia de Acessibilidade de Interfaces Web para Pessoas com Autismo (GAIA). Trata-se de um conjunto de recomendações que visa auxiliar desenvolvedores de softwares e educadores digitais a compreender como desenvolver websites que buscam atender às necessidades de pessoas com TEA, com foco em interfaces web.

A versão 2.1 da WCAG adicionou algumas recomendações relacionadas a deficiências cognitivas, que impactam pessoas com TEA, e a existência de alguns estudos 
http://dx.doi.org/10.5902/1984686X62649

relacionados à temática desenvolvidos em países como Reino Unido, Estados Unidos, Itália e Brasil, as diretrizes de design para facilitar a utilização de interfaces por pessoas com TEA carecem de dados empíricos (BRITTO; PIZZOLATO, 2016; ERASLAN et al., 2018; REZAE et al., 2020).

Entre os fatores que contribuem para a existência desta lacuna, estão: (i) o custo e complexidade necessários para pesquisar, informar, avaliar e implantar um design acessível (PAVLOV, 2014; DATTOLO; LUCCIO; PIRONE, 2016); (ii) as elaborações das diretrizes internacionais são, de forma geral, pautadas em critérios diagnósticos e previsibilidade de possíveis barreiras a serem enfrentadas, sem levar em conta a participação do usuário com TEA (RAYMAKER et al., 2019); (iii) muitas diretrizes são restritas a artigos científicos e podem apresentar linguagem de difícil compreensão (BRITTO; PIZZOLATO, 2016); (iv) grande parte das diretrizes não foi avaliada empiricamente (RAYMAKER et al., 2019; VALENCIA et al., 2019); (v) o desconhecimento dos desenvolvedores sobre as características e necessidades das pessoas com deficiências cognitivas, grupo no qual se encontram as pessoas com TEA (BRITTO; PIZZOLATO, 2016; DATTOLO; LUCCIO; PIRONE, 2016; PICHILIANI; PIZZOLATO, 2019); (vi) os estudos experimentais relativos a estas diretrizes ainda se encontram em estágios iniciais e apresentam resultados preliminares e que necessitam de maiores aprofundamentos, apesar de apresentarem contribuições relevantes para a área (YANEVA et al., 2018; RAYMAKER et al., 2019; ERASLAN et al., 2018, 2020; REZAE et al., 2020); (vii) condições de operacionalização do design universal (BRASIL, 2016).

Assim sendo, o objetivo do trabalho foi mapear na literatura pesquisas empíricas que avaliaram diretrizes para acessibilidade digital, envolvendo pessoas com TEA. Esperou-se, com este estudo, traçar uma lista de itens validados empiricamente como fundamentais para a garantia da acessibilidade digital para todas as pessoas.

\section{Método}

O presente estudo foi desenvolvido a partir de uma Revisão Integrativa (RI) da literatura. Este método tem como objetivo agregar resultados de pesquisas de maneira sistemática a respeito de uma temática determinada. Ele permite compilar, de forma sintética, diferentes estudos, com delineamentos distintos, publicados em um período de tempo específico, possibilitando um aprofundamento sobre o tema proposto, reduzindo incertezas e evidenciando lacunas, bem como sugerindo estudos posteriores como forma 
http://dx.doi.org/10.5902/1984686X62649

de densificar dados já existentes ou preencher lacunas relevantes (WHITTEMORE; KNAFL, 2005; MENDES; SILVEIRA; GALVÃO, 2008; ERCOLE; MELO; ALCOFORADO, 2014).

\section{Procedimento de coleta dos artigos}

Para realização do estudo, foram garantidos os seguintes passos: a) identificação do tema e seleção da hipótese: o tema do estudo versou sobre acessibilidade digital e TEA e esperou-se, com isso, identificar elementos necessários para a garantia da acessibilidade, via desenho universal; b) estabelecimento da estratégia de pesquisa: foram propostos logo após os critérios de inclusão e exclusão para seleção dos estudos; c) definição e coleta de dados, em três bancos (Scopus, Web of Science e Google Scholar); d) análise dos dados coletados; e) interpretação dos dados com base nos objetivos e na literatura consultada; f) apresentação dos resultados, com base na análise realizada (WHITTEMORE; KNAFL, 2005). A revisão do processo baseou-se nas recomendações da lista de conferência Preferred Reporting Items for Systematic Reviews and Meta-Analyses (PRISMA).

Figura 1 - Fluxograma da seleção dos artigos elaborado a partir das recomendações PRISMA

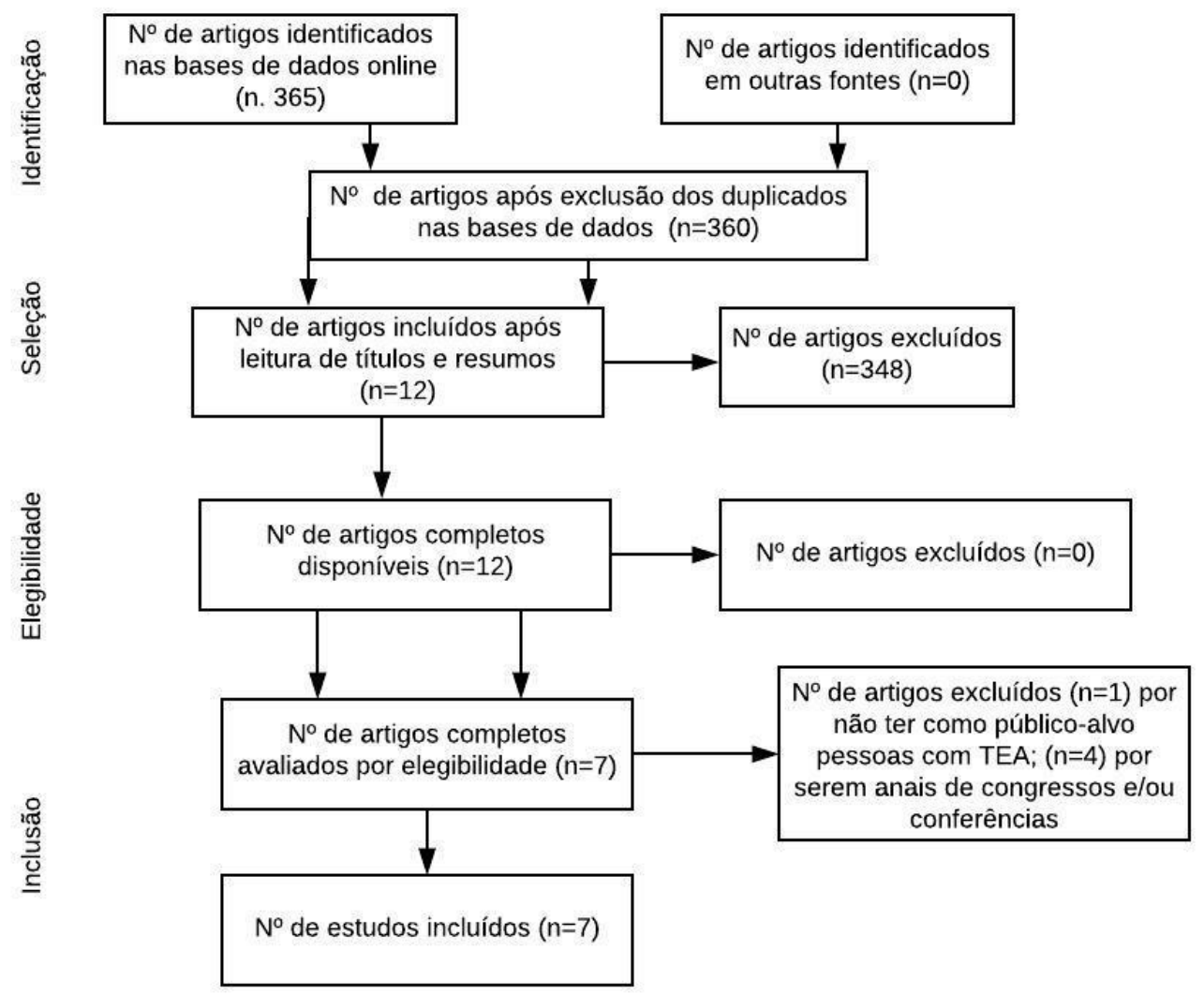

Fonte: Autoria própria (2020). 
http://dx.doi.org/10.5902/1984686X62649

O levantamento do material se deu nos meses de abril e maio de 2020. A busca foi realizada por meio de acesso remoto ao conteúdo assinado, a partir do acesso CAFE Comunidade Acadêmica Federada, provido pela Rede Nacional de Ensino e Pesquisa (RNP) para instituições federadas, a partir da Universidade Federal de São Carlos. Buscou-se por artigos indexados junto às bases de dados online Scopus, Web of Science e Google Scholar. Para as buscas, foram utilizadas as seguintes combinações de descritores: "Accessibility Guidelines" AND "Autism Spectrum Disorder" OR "Autism"; "Accessibility" AND "Autism spectrum disorder" AND "Software" OR "App"; "Guidelines Accessibility" AND "Autism"; "Accessibility Software" AND "Autism Spectrum Disorder" OR "Autism" e seus equivalentes em português e espanhol.

Foram aplicados filtros de busca conforme os critérios de inclusão e exclusão determinados. Estabeleceu-se como critérios de inclusão: a) artigos publicados nos últimos 10 anos (2010-2020); b) publicações em português, inglês ou espanhol; c) pesquisas envolvendo análises de diretrizes de acessibilidade para pessoas com autismo; d) artigos revisados por pares. Como critérios de exclusão foram considerados: a) idiomas diferentes de português, inglês ou espanhol; b) pesquisas envolvendo diretrizes de acessibilidade para outros tipos de deficiência; c) pesquisas com abordagem médica; d) teses e dissertações; e) artigos indisponíveis (não encontrados).

\section{Análise dos dados}

A análise foi estruturada em dois eixos. O primeiro sobre as principais características dos estudos em relação ao ano de publicação, revista, país e participantes. A segunda análise foi qualitativa sobre as diretrizes utilizadas, os principais resultados e os itens mensurados como fundamentais para garantia da acessibilidade digital das pessoas com TEA, de acordo com os estudos recuperados com a busca.

\section{Resultados}

$\mathrm{Na}$ busca inicial junto às bases de dados utilizando as combinações de descritores supra e retro apresentados, foram encontradas 365 publicações, sendo 42 na base de dados Scopus, 16 na base Web of Science e 307 na base Google Scholar. Em seguida, foram identificados cinco artigos duplicados (o mesmo estudo ocorreu em mais de uma base de dados), restando 360 artigos. Após a leitura exploratória dos títulos e resumos, foram previamente selecionados 12 artigos e excluídos 348. Foi realizada a leitura 
analítica integral destes 12 artigos, sendo que, após a aplicação dos critérios de elegibilidade, sete foram incluídos para compor o corpus da revisão e cinco foram excluídos (um por não ter como público-alvo pessoas com TEA e quatro por serem publicações realizadas em anais de congressos e/ou conferências) (vide Figura 1).

Dos sete artigos que compuseram o corpus desta revisão, seis foram publicados em periódicos internacionais e um em periódico brasileiro. No que concerne ao ano de publicação, embora tenham sido buscados, não foram encontrados estudos que atendessem aos critérios de inclusão entre 2010 e 2016. Entre os artigos incluídos, um artigo foi publicado em 2017, três em 2018, um em 2019 e dois em 2020. Foi possível perceber uma diversidade cultural entre os trabalhos publicados, contando com contribuições do Reino Unido (3), Itália (1), Estados Unidos (1), Austrália (1) e Brasil (1) (vide Figura 2).

Com relação aos participantes/público-alvo das pesquisas, a maioria dos trabalhos teve como participantes pessoas adultas diagnosticadas com TEA leve (3 - ERASLAN et al., 2018, 2020; REZAE et al., 2020), dois estudos com adultos com TEA (RAYMAKER et al., 2019; YANEVA et al., 2018). Um estudo envolveu participantes cuidadores responsáveis pelo suporte a pessoas com TEA entre 3 e 12 anos e profissionais da tecnologia da informação (BRITTO; PIZZOLATO, 2018). Um estudo com crianças com TEA (DATTOLO; LUCCIO; PIRONE, 2016); todavia, tal estudo não envolveu testagens experimentais, apenas elaboração de diretrizes de acessibilidade e análise de sites e aplicativos com base nessas diretrizes. É fundamental ressaltar que tais pesquisas não envolveram crianças, adolescentes e adultos com TEA moderado ou severo (Figura 2). 
http://dx.doi.org/10.5902/1984686X62649

Figura 2 - Características gerais dos estudos considerados como resultados

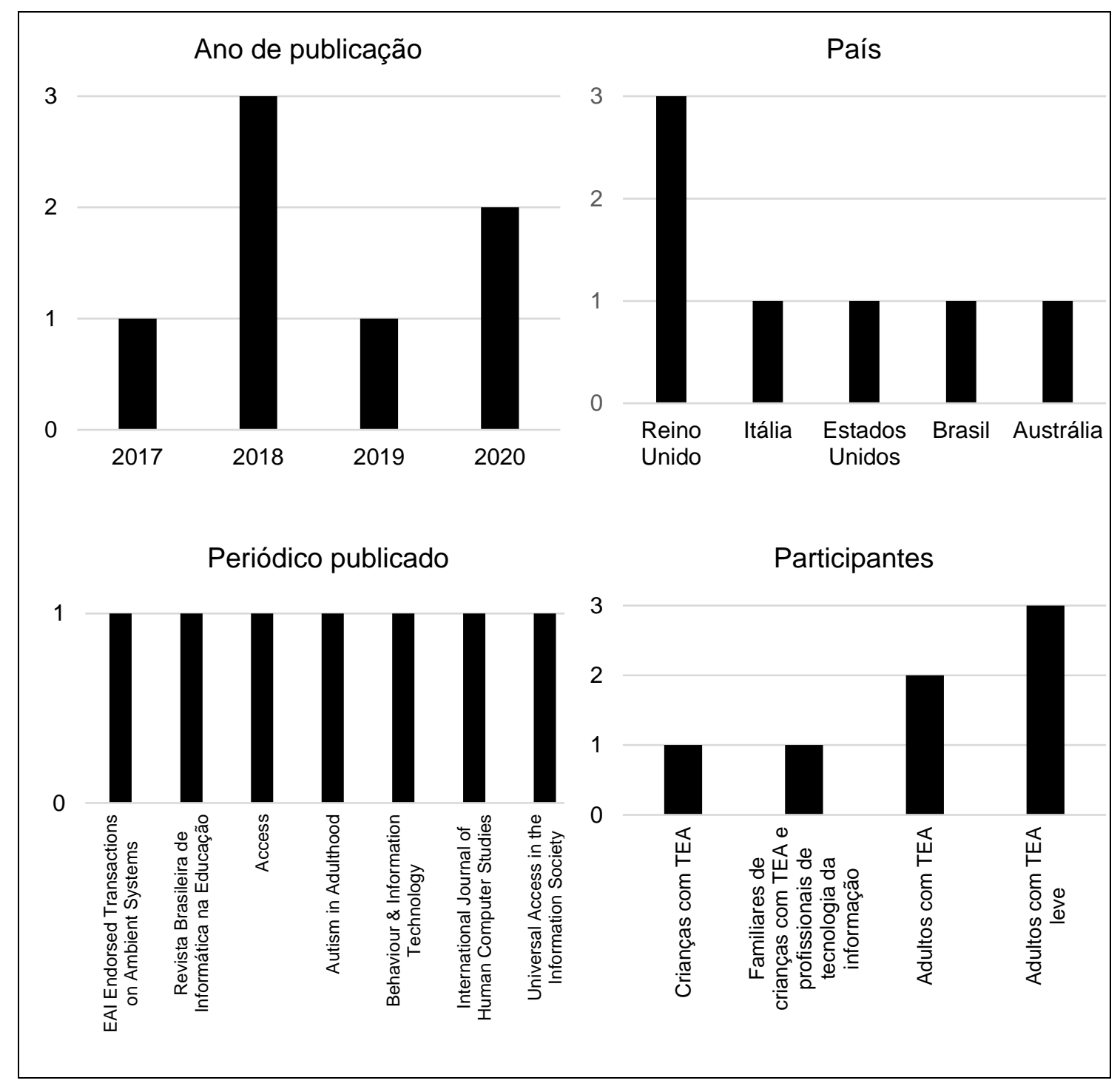

Fonte: Autoria própria (2020).

Ainda sobre os participantes, é fundamental ressaltar que os estudos conduzidos com adultos com TEA leve realizaram análises de acessibilidade a partir de estudos empíricos experimentais, utilizando a técnica de rastreamento ocular e presença de grupo controle com pessoas sem TEA. Os dados obtidos foram analisados estatisticamente (YANEVA et al., 2018; ERASLAN et al., 2018, 2020; REZAE et al., 2020), com comparações entre grupos. O estudo comparativo analisou a adequação de sites e aplicativos às diretrizes de acessibilidade desenvolvidas pelos autores (DATTOLO; LUCCIO; PIRONE, 2016). O estudo participativo, a partir da análise de diretrizes de acessibilidade já existentes, elaborou um conjunto de diretrizes para apoiar a elaboração de um site para saúde voltado para pessoas com TEA e testes de avaliação junto a pessoas no espectro e cuidadores (RAYMAKER et al., 2019). Por fim, o estudo bibliográfico exploratório se deu mediante uma 
http://dx.doi.org/10.5902/1984686X62649

exaustiva revisão de literatura a diretrizes, estudos e práticas de acessibilidade envolvendo pessoas com TEA, e também com deficiências cognitivas, compilando as recomendações mais recorrentes que embasaram a elaboração das diretrizes voltadas para o público-alvo do estudo e avaliação piloto das recomendações (BRITTO; PIZZOLATO, 2018).

Os estudos valeram-se das diretrizes internacionais estabelecidas pela W3C sobre acessibilidade para embasar suas análises, seja a partir de experimentação, estudos comparativos, ou mesmo propositura de diretrizes próprias para pessoas com TEA. O Quadro 1 mostra as diretrizes e os principais resultados dos estudos.

Quadro 1 - Estudos sobre avaliação de diretrizes de tecnologia digital e o TEA

\begin{tabular}{|c|c|c|}
\hline Diretriz & Tema da diretriz & Principais resultados \\
\hline $\begin{array}{l}\text { Elaboração } \\
\text { própria }\end{array}$ & $\begin{array}{l}\text { Acessibilidade e } \\
\text { usabilidade para sites e } \\
\text { aplicativos móveis para } \\
\text { usuários com TEA } \\
\text { (DATTOLO; FLAMINIA, } \\
\text { 2017). }\end{array}$ & $\begin{array}{l}\text { Sites: Maioria direcionada a usuários com TEA leve } \\
\text { adultos. A capacidade de resposta é negligenciada, não } \\
\text { há subdivisão de tarefas e adaptação. } \\
\text { Apps: Limitações de navegação e previsibilidade das } \\
\text { tarefas. Apesar de possibilitarem a personalização, esta } \\
\text { é muitas vezes complexa de ser realizada. Uso de } \\
\text { sintetizadores disponíveis em diferentes aplicações. } \\
\text { Produção de sons que não são facilmente reconhecidos } \\
\text { por usuários. A adaptação da linguagem e também das } \\
\text { vozes melhoraria muito a qualidade dessas aplicações. }\end{array}$ \\
\hline $\begin{array}{l}\text { Elaboração } \\
\text { própria }\end{array}$ & $\begin{array}{l}\text { Desenvolvimento de } \\
\text { websites adequados às } \\
\text { necessidades de crianças } \\
\text { com TEA, em plataforma } \\
\text { web e/ou dispositivos } \\
\text { multitoque (BRITTO; } \\
\text { PIZZOLATO, 2018). }\end{array}$ & $\begin{array}{l}\text { Vocabulário visual e textual: linguagem simples, sem } \\
\text { ironia, jargões, abreviações, frases curtas, uso de } \\
\text { marcadores como listas, representação de ações no } \\
\text { cotidiano, etc. } \\
\text { Engajamento: interface simples, com poucos elementos, } \\
\text { separar conteúdos distintos, etc. } \\
\text { Customização: cores, controle de usuário, texto, fontes, } \\
\text { etc. }\end{array}$ \\
\hline $\begin{array}{l}\text { Elaboração } \\
\text { própria }\end{array}$ & $\begin{array}{l}\text { Desenvolvimento de site de } \\
\text { saúde acessível destinado } \\
\text { a usuários com TEA da } \\
\text { web (RAYMAKER et al., } \\
\text { 2019). }\end{array}$ & $\begin{array}{l}3 \text { categorias, diretrizes de acessibilidade física, } \\
\text { acessibilidade intelectual e acessibilidade social. }\end{array}$ \\
\hline $\begin{array}{l}\text { Avaliação de } \\
\text { diretrizes de } \\
\text { acessibilidade, } \\
\text { relacionadas à } \\
\text { complexidade } \\
\text { visual de web } \\
\text { sites com uso } \\
\text { de eye } \\
\text { tracking }\end{array}$ & $\begin{array}{l}\text { Complexidade visual da } \\
\text { página da web e } \\
\text { capacidade de } \\
\text { diferenciação dos } \\
\text { elementos das páginas da } \\
\text { web (ERASLAN et al., } \\
\text { 2020). }\end{array}$ & $\begin{array}{l}\text { Comportamento diferente do grupo de pessoas com e } \\
\text { sem TEA, na realização de tarefas específicas na web. } \\
\text { Sites com layouts muito complexos dificultam a } \\
\text { interação de pessoas com TEA, pois tendem a focar em } \\
\text { elementos irrelevantes das páginas visualmente } \\
\text { complexas ou nas quais os elementos não estão } \\
\text { facilmente distinguíveis. A alta complexidade visual de } \\
\text { uma página ou a baixa diferenciação de seus elementos } \\
\text { provoca efeitos diferentes entre grupos de pessoas com } \\
\text { e sem TEA. }\end{array}$ \\
\hline
\end{tabular}

Fonte: Autores do presente estudo (2020).

No que diz respeito aos objetivos dos estudos, cinco deles buscaram avaliar a interação das pessoas com TEA nos ambientes virtuais (webpages), a fim de verificar 
http://dx.doi.org/10.5902/1984686X62649

possíveis diferenciações entre os caminhos utilizados pelas pessoas com e sem TEA na busca por informações na web, de modo a testar os fundamentos que direcionam as diretrizes de acessibilidade internacionais (YANEVA et al., 2018; ERASLAN et al., 2018, 2020; REZAE et al., 2020).

Outra pesquisa realizou uma extensa revisão de trabalhos envolvendo diretrizes e práticas de acessibilidade recomendadas para elaboração e interfaces para pessoas com TEA e outras recomendações internacionais, a fim de verificar as de maior incidência e propor um conjunto de diretrizes próprias que contemple as especificidades de usuários com TEA, especialmente crianças; também houve entrevistas com familiares e testagem piloto das recomendações por especialistas (BRITTO; PIZZOLATO, 2018). Um estudo buscou o desenvolvimento, avaliação e validação de site para contribuir nos cuidados com a saúde voltados a pessoas com TEA, avaliado por meio de testes aplicados com pessoas com TEA e cuidadores (RAYMAKER et al., 2019); um avaliou sites disponibilizados para crianças com TEA, a fim de verificar se os mesmos atendem às recomendações de acessibilidade estabelecidas para este público, desenvolvidas pelos autores (DATTOLO; LUCCIO; PIRONE, 2016). O Quadro 2 reuniu as características digitais analisadas pelos estudos, com uso de delineamento experimental de grupo de pessoas com e sem TEA.

Quadro 2 - Características digitais analisadas nos estudos com delineamento de grupo de pessoas com e sem TEA

\begin{tabular}{|c|c|c|}
\hline \multirow{4}{*}{ 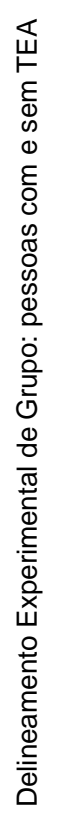 } & Características digitais & Resultados \\
\hline & $\begin{array}{l}\text { Processamento de páginas da } \\
\text { web, com foco na atenção visual } \\
\text { (YANEVA et al., 2018). }\end{array}$ & $\begin{array}{l}\text { Os participantes com TEA focaram mais em elementos } \\
\text { irrelevantes (que apresentam objetivo periférico ou que não } \\
\text { eram relevantes para a tarefa), enquanto estes foram } \\
\text { descartados pelo grupo controle ao executar as tarefas. Os } \\
\text { dados revelaram ainda que, mesmo após terem encontrado a } \\
\text { resposta correta, usuários com TEA continuaram a pesquisar, } \\
\text { o que traz evidência de que não reconheceram a resposta } \\
\text { como correta. }\end{array}$ \\
\hline & $\begin{array}{l}\text { Busca por informações em } \\
\text { páginas da internet, com } \\
\text { enfoque na distração causada } \\
\text { por conteúdo secundário e } \\
\text { confusão causada por transições } \\
\text { entre os elementos de páginas } \\
\text { da web (ERASLAN et al., 2019). }\end{array}$ & $\begin{array}{l}\text { Pessoas com TEA: olharam mais para elementos irrelevantes } \\
\text { e tiveram verificação de caminhos mais longos. O tempo de } \\
\text { duração das fixações foi menor, realizaram maiores } \\
\text { transações entre elementos das páginas da web, } \\
\text { apresentando variações dentro do próprio grupo, com uso de } \\
\text { diferentes estratégias de busca de informações quando } \\
\text { comparadas a pessoas sem TEA. }\end{array}$ \\
\hline & $\begin{array}{l}\text { Avaliar um aplicativo de } \\
\text { transporte público desenvolvido } \\
\text { a partir de recomendações de } \\
\text { acessibilidade para pessoas com } \\
\text { TEA (REZAE et al., 2020). }\end{array}$ & $\begin{array}{l}\text { Pessoas com TEA processam mais facilmente ícones e } \\
\text { imagens, em relação aos textos. O uso concomitante de } \\
\text { textos e ícones facilita a compreensão de usuários com TEA, } \\
\text { desde que estes sejam complementares, caso contrário, } \\
\text { confunde-os. }\end{array}$ \\
\hline
\end{tabular}

Fonte: Autores do presente estudo (2020). 
http://dx.doi.org/10.5902/1984686X62649

A técnica de rastreamento ocular foi utilizada nos estudos (ERASLAN et al., 2019; YANEVA et al., 2018) para comparação das medidas do olhar, no caso, número e tempo de fixações oculares para processamento das páginas e busca de informações em páginas da internet. Esses resultados são importantes para orientação de design digital na elaboração de páginas mais amigáveis para todas as pessoas.

\section{Discussão}

O mapeamento sistemático da literatura identificou sete pesquisas empíricas que avaliaram diretrizes para acessibilidade digital, sendo que a maioria ( $n=3$ - Quadro 1) utilizou um protocolo próprio, elaborado pelos próprios autores, a partir de estudos fundamentados na literatura. Os principais resultados identificados como itens promissores na construção e/ou adequação de recursos digitais foram: a) em site existe a necessidade de subdivisão de tarefas e adaptação e em apps é preciso deixar mais previsíveis as tarefas, com tarefas personalizadas mais facilmente programáveis e adaptação da linguagem (DATTOLO; FLAMINIA, 2017); b) sites com poucos layouts (ERASLAN et al., 2020), c) uso de ícones e imagens concomitantes ao texto, de maneira completar (REZAE et al., 2020); d) vocabulário visual e textual, engajamento e customização (BRITTO; PIZZOLATO, 2018).

A partir dos estudos analisados, constatou-se que existe uma baixa produção $(n=7)$ de estudos baseados em evidências empíricas promovidas por estudos experimentais, no que diz respeito à acessibilidade de interfaces para pessoas com TEA, especialmente quando se trata de crianças. Esta carência pode estar ligada à pouca atenção destinada à acessibilidade cognitiva (BRITTO; PIZZOLATO, 2018; YANEVA et al., 2020; REZAE et al., 2020), que somente passou a ser objeto de pesquisa há pouco mais de uma década, quando Friedman e Bryen (2006) realizaram os primeiros ensaios sobre o tema. Os autores propuseram um conjunto de 22 diretrizes de acessibilidade da web para pessoas com transtornos do neurodesenvolvimento. Para tal, realizaram uma extensa revisão bibliográfica de estudos envovendo diretrizes de especialistas, governos e instituições. Os pesquisadores fizeram recomendações com relação ao uso de figuras, gráficos, ícones e símbolos junto com o texto; uso de texto claro e simples; uso de naveagação e design consistente; uso de cabeçalhos, títulos e avisos. Embora não tenham sido desenvolvidas especificamentes para o público de pessoas com TEA, estas diretrizes trouxeram implicações importantes para se pensar em guidelines voltadas para estes usuários. 
http://dx.doi.org/10.5902/1984686X62649

Outra forma de desenvolver diretrizes pode ser feita por familiares, professores e profissionais que atuam com pessoas com TEA. Putnam e Chong (2008), apesar de não apresentarem diretrizes de acessibilidade, desenvolveram um estudo empírico importante com pais e professores de pessoas com TEA, buscando destacar aspectos que podem auxiliar na criação de interfaces tecnológicas para essas pessoas, de modo a dialogar com os achados de Britto e Pizzolato (2018) para proposição das diretrizes de acessibilidade.

De maneira geral, nota-se uma curva ascendente no número de publicações (Figura 2), de acordo com a distribuição temporal dos artigos publicados ao longo do período analisado, por meio de estudos experimentais com grupos de usuários com TEA (ERASLAN et al., 2018, 2020; RAYMAKER et al., 2019; REAZE et al., 2020).

Estes achados sugerem que as pesquisas sobre acessibilidade, bem com a própria definição destas diretrizes, ainda se encontram em fase preliminar, sobretudo no escopo nacional. Outro fator importante é que, apesar das similaridades entre as diretrizes apresentas (DATTOLO; LUCCIO; PIRONE, 2016; BRITTO; PIZZOLATO, 2018; RAYMAKER et al., 2019), ainda não há uma uniformização das mesmas. Talvez pelo fato de as diretrizes de acessibilidade internacionais, como as da WCAG, listarem os requisitos dos usuários da web com TEA sob o termo geral "deficiências cognitivas". Este fator pode ser também uma das razões pelas quais ainda não haja uma preocupação efetiva com estas diretrizes.

Percebe-se que a maioria dos estudos experimentais, para coleta dos dados, utilizou a técnica de rastreamento ocular, com uso de um dispositivo para avaliar o olhar do participante. Trata-se de uma técnica que possibilita o rastreamento e a gravação dos comportamentos oculares, tais como número de fixações oculares, tempo de duração de cada fixação e respectiva velocidade, caminho percorrido pela pupila, dilatação da pupila e sacada (GREBOT, 2016). Sua utilização tem sido crescente em pesquisas que envolvem testes de usabilidade, especialmente com pessoas com TEA, uma vez que a medida do olhar se refere a uma medida de processamento visual, que necessariamente envolve a atenção (RODAS; MARCOS; VIDOTTI, 2014). Apesar da eficiência apresentada nos estudos, esta técnica pode apresentar algumas inconsistências, uma vez que a contagem pura de fixações pode não revelar a real interação dos usuários com a interface. Alguns desafios ainda são postos com o uso desta técnica, como calibração, uso com crianças pequenas, uso de dispositivo móvel etc. (NIELSEN; PERNICE, 2009). 
Outro aspecto importante a se destacar é que as pesquisas experimentais foram realizadas, em sua maioria, com adultos com TEA leve. Isto se apresenta como uma limitação e um viés, já que resultados diferentes podem ser encontrados em pesquisas conduzidas com pessoas com TEA com outras idades e, também, outros níveis de acometimento dos sintomas. Este fato impossibilita a generalização desses estudos.

Pessoas com TEA podem apresentar diferenças de processamento visual se comparadas às pessoas sem TEA, entre elas estão disfunção executiva, coerência central e em alguns casos, baixa velocidade de processamento (HAPPÉ; FRITH, 2006; MINTZ, 2013). A disfunção executiva faz com que pessoas com TEA encontrem dificuldades em iniciar tarefas e identificar as etapas necessárias para cumpri-las (MINTZ, 2013). Indivíduos com pouca coerência central podem se concentrar excessivamente nos detalhes de um cenário e terem dificuldades para entender o significado geral (BOOTH; HAPPÉ, 2010; MINTZ, 2013). Assim, a forma com que a informação é apresentada às pessoas com TEA pode influenciar no modo como elas irão interagir com a mesma (PAVLOV, 2014; ERASLAN et al., 2018; YANEVA; TEMINIKOVA; MITKOV, 2015).

Nos estudos que compuseram esta revisão, verificou-se que sites com layout e linguagens complexos, textos longos, com muitas transições entre os elementos da tela, podem se tornar desafiadores para qualquer sujeito, pois requerem um esforço cognitivo mais elevado em relação à realização de tarefas simples (YANEVA et al., 2018; ERASLAN et al., 2018).

Estes dados corroboram a importância de garantir um designer de forma explícita e simples, sem muitos elementos distratores, a fim de facilitar o entendimento das informações fornecidas (DATTOLO; LUCCIO; PIRONE, 2016; BRITTO; PAVLOV, 2014; PIZZOLATO, 2018; RAYMAKER et al., 2019).

Testando empiricamente sites com diferentes níveis de complexidade em relação ao layout, Eraslan et al. $(2019,2020)$ constataram que pessoas com TEA apresentam mais dificuldade de realizar tarefas em sites com visual complexo. Isto porque tendem a se fixar em elementos irrelevantes quando realizam buscas na web. Esta fixação em elementos irrelevantes também foi percebida por Yaneva et al. (2018) e Rezae et al. (2020).

Outros aspectos relativos ao design da interface, como desafios na capacidade de resposta, ausência de subdivisão e previsibilidade de tarefas, utilização de ícones ambíguos ou incomuns, foram apontados como fatores que influem de forma negativa para 
a interação de pessoas com TEA em interfaces digitais (DATTOLO; LUCCIO; PIRONE, 2016; REZAE et al., 2020).

Uma estratégia promissora tem sido a discussão acerca da garantia do design universal, por meio da personalização dos itens disponíveis nas plataformas digitais. Este foi um meio identificado em um dos estudos (DATTOLO; FLAMINIA, 2017) como promissores para operacionalização de diretrizes que atendam a um número maior de pessoas, independentemente de suas condições. Apesar dos desafios relacionados à garantia de uma personalização que ocorra com interface amigável para leigos na área computacional, é um passo importante para garantia do design universal previsto na legislação nacional (BRASIL, 2016). A partir da personalização, o usuário com deficiência ou transtorno torna-se protagonista das escolhas que realiza para acessar o conteúdo digital, por exemplo, pessoas com baixa visão que determinam o uso de cores com alto contraste podem não ser adequadas para as necessidades de usuários com TEA com visão hipersensível (RAYMAKER et al., 2019), então a personalização da ferramenta garante que o usuário, enquanto protagonista, defina qual o melhor recurso para uso da ferramenta digital.

É cediço que o autismo é considerado um espectro em virtude da diversidade existente entre as pessoas. Apesar da existência de um repertório comum à maioria dos indivíduos, este não é suficiente para caracterizar as pessoas com TEA como um grupo homogêneo. Diferenças individuais no que diz respeito ao nível de desenvolvimento, habilidades, prejuízos orgânicos, fatores socioeconômicos, culturais, entre outras, podem ser encontradas entre as pessoas que se encontram no espectro (WINDHOLZ, 1995).

Assim, embora a elaboração de diretrizes que visem facilitar a interação de pessoas com TEA seja de grande valia, não é possível, pelo menos por ora, afirmar que elas sejam capazes de contemplar interação de pessoas com diferentes níveis de TEA com recursos informatizados. Até mesmo porque faltam evidências empiricamente testadas para comprovar sua eficácia com crianças, adolescentes, idosos com TEA ou com outras comorbidades, uma vez que as pesquisas aqui apresentadas relatam apenas a experiência de uma amostra composta por parte da população com TEA leve, o que significa que não há déficit cognitivo e maior autonomia, em relação ao baixo uso de suportes.

O que se tem até o momento, pelo menos quanto a hipóteses, é que as diretrizes de acessibilidade voltadas para usuários com TEA têm possibilitado ofertar maior 
http://dx.doi.org/10.5902/1984686X62649

acessibilidade a eles, principalmente porque possibilitam a personalização e simplicidade das interfaces.

Estudos avaliando empiricamente estas diretrizes com usuários com TEA pertencentes a outros níveis podem trazer resultados diferentes dos encontrados. É necessário, ainda, pensar que contextos sociais e culturais também podem influenciar na forma com que o usuário interage com a interface, até mesmo porque os estudos experimentais foram conduzidos em países cuja tecnologia se apresenta em estágios bastante avançados.

\section{Considerações finais}

Esperou-se, com este estudo, traçar uma lista de itens validados empiricamente como fundamentais para garantia da acessibilidade digital para todas as pessoas, sobretudo para pessoas com TEA. Atualmente, os princípios de design associados ao design de interação para os transtornos do neurodesenvolvimento ainda são generalistas e carecem de maiores testes com públicos diferenciados, como crianças e adolescentes.

Diretrizes de acessibilidade voltadas para usuários com TEA ainda se encontram em estágios iniciais de elaboração, apesar do número expressivo de aplicativos, softwares e webpages que se intitulam como destinados ao público com TEA, disponíveis nas lojas virtuais de aplicativos.

Outro fator a ser considerado é que as diretrizes de acessibilidade para pessoas com TEA elaboradas ainda carecem de evidências que comprovem a sua efetividade, sobretudo em relação ao número de testes realizados e o perfil comportamental dos usuários. Eis que a maioria delas ainda não foi testada empiricamente. Estudos buscando avaliar estas diretrizes são de grande valia para que se possam elaborar aplicativos, sites e softwares que realmente promovam a acessibilidade e a interação de forma independente por pessoas com TEA.

Pesquisas experimentais analisando as funcionalidades das diretrizes de acessibilidade para pessoas com TEA são necessárias diante da carência de estudos existentes na literatura. Além do mais, os poucos estudos empíricos experimentais existentes não podem ser generalizados para todos os níveis de acometimento do TEA, razão pela qual devem ser conduzidas outras pesquisas, a fim de fornecer mais evidências empíricas sobre a efetividade destas diretrizes. 
http://dx.doi.org/10.5902/1984686X62649

\section{Referências}

APA - American Psychiatric Association. Manual Diagnóstico e Estatístico de

Transtornos Mentais - DSM-5. Tradução de Maria Inês Corrêa Nascimento et al. Porto Alegre: Artmed, 2014.

BRASIL. Lei Brasileira de Inclusão. 2016. Disponível em:

http://www.planalto.gov.br/ccivil_03/_ato2015-2018/2015/lei/l13146.htm Acesso em: 28 out. 2020.

BOOTH, Rhonda; HAPPÉ, Francesca. 'Hunting with a knife and... fork': Examining central coherence in autism, attention deficit/hyper-activity disorder, and typical development with a linguistic task. Journal of Experimental Child Psychology, v. 107, n. 4, p. 377-393, nov. 2010.

BRITTO, Talita Cristina Pagani; PIZZOLATO, Ednaldo Brigante. Towards Web Accessibility Guidelines of Interaction and Interface Design for People with Autism Spectrum Disorder. In: THE NINTH INTERNATIONAL CONFERENCE ON ADVANCES IN COMPUTER-HUMAN INTERACTIONS, 2016, Veneza. Anais eletrônicos... Veneza: ACHI, 2016. Disponível em: https://www.researchgate.net/publication/301552021_Towards Web_Accessibility_Guidelines_of_Interaction_and_Interface_Design_for_People_with_Aut ism_Spectrum_Disorder. Acesso em: 18 maio 2020.

BRITTO, Talita; PIZZOLATO, Ednaldo Brigante. GAIA: uma proposta de um guia de recomendações de acessibilidade de interfaces Web com foco em aspectos do Autismo. Revista Brasileira de Informática na Educação - RBIE, v. 26, n. 2, maio 2018.

CHIEN, Miao En et al. iCAN: a tablet-based pedagogical system for improving communication skills of children with autism. International Journal of Human-Computer Studies, v. 73, p. 79-90, jan. 2015.

DAREJEH, Ali; SINGH, Dalbir. A review on user interface design principles to increase software usability for users with less computer literacy. Journal of Computational Sciense, n. 9, p. 1443-1450, out. 2013.

DATTOLO, Antonina; LUCCIO, Flaminia L.; PIRONE, Elisa. Webpage Accessibility and Usability for Autistic Users: a Case Study on a Tourism Website. In: THE NINTH INTERNATIONAL CONFERENCE ON ADVANCES IN COMPUTER-HUMAN INTERACTIONS, 2016, Veneza. Anais eletrônicos... Veneza: ACHI, 2016. Disponível em: https://core.ac.uk/reader/80136806. Acesso em: 18 maio 2020.

DATTOLO, Antonina; LUCCIO, Flaminia L. Accessible and usable websites and mobile applications for people with autism spectrum disorders: a comparative study. EAI Endorsed Transactions on Ambient Systems v. 4, n.13. p. 1-11, maio. 2017.

ERASLAN, Sukru. et al. Web users with autism: Eye tracking evidence for differences. Behaviour and Information Technology, v. 38, n. 7, p. 678-700, dez. 2018. 
ERASLAN, Sukru. et al. Web users with autism: Eye tracking evidence for differences. Behaviour and Information Technology, v.38, n.7, p.678-700, 2019.

ERASLAN, Sukru. et al. "Keep it simple!": An eye-tracking study for exploring complexity and distinguishability of web pages for people with autism. Universal Access in the Information Society, p. 1-16, fev. 2020.

ERCOLE, Flávia Falci; MELO, Laís Samara de; ALCOFORADO, Carla Lúcia Goulart Constant. Revisão integrativa versus revisão sistemática. Revista Mineira de Enfermagem, v. 18, n. 1, p. 9-12, 2014.

FRIEDMAN, Mark G.; BRYEN, Diane Nelson. Web accessibility design recommendations for people with cognitive disabilities. Technology and Disability, v. 19, n. 4, p. 205-212, dez. 2006.

GREBOT, Ivan Bouchardet da Fonseca. Padrões de rastreamento na visualização de faces próprias e não próprias. 2016. 48 f. Dissertação (Mestrado em Ciências do Comportamento) - Universidade de Brasília, Brasília, 2016.

HAPPÉ, Francesca; FRITH, Uta. The Weak Coherence Account: Detail Focused Cognitive Style in Autism Spectrum Disorder. Journal of Autism and Developmental Disorders, $n$. 36, p. 5-25, 2006.

HENRY, Shawn Lawton. Just Ask: Integrating Accessibility throughhout Design. Lulu.com, 2007.

LOCATELLI, Paula Borges; SANTOS, Mariana Fernandes Ramos dos Santos. Autismo: Propostas de intervenção. Revista Transformar, n. 8, p. 203-220, 2016.

MEDAVARAPU, Srinivas. et al. Where is the Evidence? A Narrative Literature Review of the Treatment Modalities for Autism Spectrum Disorders. Cureus, v. 11, n. 1, p. e3901, 2019. Disponível em: http://dx.doi.org/10.7759/cureus.3901. Acesso em: 18 maio 2020.

MENDES, Karina Dal Sasso; SILVEIRA, Renata Cristina de Campos Pereira; GALVÃO, Cristina Maria. Revisão integrativa: método de pesquisa para a incorporação de evidências na saúde e na enfermagem. Texto \& Contexto Enfermagem, Florianópolis, v. 17, n. 4, p. 758-764, dez. 2008.

MINTZ,Joseph. Additional Key Factors Mediating the Use of a Mobile Technology Tool Designed to Develop Social and Life Skills in Children with Autism Spectrum Disorders: Evaluation of the 2nd HANDS Prototype. Computers \& Education, v. 63, p. 17-27, abr. 2013.

MOTTRON, Laurent. et al. Enhanced perceptual functioning in autism: an update, and eight principles of autistic perception. Journal of Autism and Developmental Disorders, v. 36, n. 1, p. 27-43, jan. 2006.

NIELSEN, Jacob; PERNICE, Kara. Eye tracking Web Usability. Berkeley, CA, USA, 2009. 
PAVLOV, Nikolay. User Interface for People with Autism Spectrum Disorders. Journal of Software Engineering and Applications, n. 7, p. 128-134, fev. 2014.

PICHILIANI, Talita Cristina Pagani Britto; PIZZOLATO, Ednaldo Brigante.

A survey on the awareness of brazilian web development community about cognitive accessibility. In: 18TH BRAZILIAN SYMPOSIUM ON HUMAN FACTORS IN COMPUTING SYSTEMS, Vitória, Espírito Santo, Brazil, 2019. Proceedings... Vitória: Association for Computing Machinery, 2019. p. 1-11.

PUTNAM, Cynthia.; CHONG Lorna. Software and technologies designed for people with autism: what do users want? In: 10TH INTERNATIONAL ACM SIGACCESS CONFERENCE ON COMPUTERS AND ACCESSIBILITY, Halifax, Nova Scotia, Canada, 2008. Proceedings... Halifax, 2008. p. 13-15.

RAYMAKER, Dora M. et al. Development of the AASPIRE Web Accessibility Guidelines for Autistic Web Users.. Autism in Adulthood. v.1, n.2, p. 146-157, 2020.

REZAE, Mortaza. et al. The evaluation of a mobile user interface for people on the autismo spectrum: An eye movement study. International Journal of Human-Computer Studies, v. 142 , p. 1-35, 2020.

RODAS, Cecilio Merlotti; MARCOS, Mari-Carmen; VIDOTTI, Silvana Aparecida Borsetti Gregório. Tecnologia de Eye Tracking em User Experience. In: ENCONTRO NACIONAL DE GESTÃO, POLÍTICAS E TECNOLOGIAS DE INFORMAÇÃO, 2014, Goiânia. Anais... Goiânia: UFG, 2014. p. 1-8.

SEEMAN, Lisa; COOPER, Michael. Cognitive Accessibility Roadmap and Gap Analysis. Retrieved June 27, 2016. Disponível em: http://w3c.github.io/coga/gap-analysis/ Acesso em: 22 jul. 2020.

SILVA, Bruno Santana da; BARBOSA, Simone Diniz Junqueira. Interação HumanoComputador: Projetando a Experiência Perfeita. Rio de Janeiro: Campus, 2010.

SILVA, M. D. da; SOARES, A. C. B.; BENITEZ, P. Software mTEA: do Desenho Computacional à Aplicação por Profissionais com Estudantes com Autismo. Revista Brasileira de Educação Especial, v. 26, n. 1, p. 51-68, 2020.

TREVISAN, D. et al. A Review of the Use of Computational Technology in Applied Behavior Analysis. Adaptive Behavior, v. 27, p. 183-196, 2019.

VALENCIA, Katherine. et al. The Impact of Technology on People with Autism Spectrum Disorder: A Systematic Literature Review. Sensors (Basel), v. 19, n. 20, p. 4485, out. 2019.

WINDHOLZ, Margarida H. Autismo infantil: terapia comportamental. In: SCHWARTZMAN, José Salomão; ASSUMPÇÃO, Francisco Baptista Júnior. (Org.). Autismo infantil. São Paulo: Memnon, 1995. p. 179-210.

WHITTEMORE, Robin; KNAFL, Kathleen. The integrative re-view: updated methodology. Journal of Advanced Nursing, v. 52, n. 5, p. 546-553, 2005. 
YANEVA, Victoria; TEMINIKOVA, Irina; MITKOV, Ruslan. Accessible Texts for Autism: An Eye-Tracking Study. In: THE 17TH INTERNATIONAL ACM SIGACCESS CONFERENCE OF COMPUTERS AND ACCESSIBILITY, Lisboa, Portugal, 2015. Anais... Lisboa, 2015. p. 49-57.

YANEVA, Victoria. et al. Autism and the web: using web-searching tasks to detect autism and improve web accessibility. ACM SIGACCESS Accessibility and Computing, v. 121, n. 2, ago. 2018. Disponível em: https://dl.acm.org/newsletter/sigaccess. Acesso em: 18 maio 2020.

\section{Notas}

${ }^{1}$ Consórcio de empresas que desenvolve e mantém os padrões de tecnologia sobre a web.

(c) (7) (9) International (CC BY-NC 4.0) 